\title{
Formation of Anthocyanin Ion-pairs. A Co-Pigmentation Effect
}

\author{
Paulo Figueiredo ${ }^{*, a}$ and Fernando Pina ${ }^{a, b}$ \\ a Departamento de Química da Faculdade de Ciências e Tecnologia da Universidade Nova de Lisboa, \\ Quinta da Torre 2825-Monte de Caparica, Portugal \\ b Instituto de Tecnologia Química e Biológica, Oeiras, Portugal
}

\begin{abstract}
The influence of the addition of ionic salts $\left(\mathrm{NaCl}, \mathrm{NaBr}\right.$. Nal. $\left.\mathrm{NaClO}_{4}\right)$ on anthocyanin equilibria is studied in this work. The effect of their addition is a colour enhancement through the formation of an ion-pair between the charged flavylium cation and the anion, which displaces the equilibria towards the coloured flavylium form. The ion-pair formation is an alternative model to that which suggests a preferable hydration of salt anions instead of the flavylium cation. Throughout this work two natural anthocyanins are studied (malvidin 3,5-diglucoside and malvidin 3-glucoside) as well as one anthocyanidin (malvidin). The thermal and photochemical stability of the ion-pairs formed is also examined.

Experiments with a synthetic anthocyanin analogue (4',7-dihydroxyflavylium chloride) which has photochromic properties are also described for comparison purposes.
\end{abstract}

Anthocyanins are very interesting natural colourants, responsible for most of the red, blue and purple colours of flowers and fruits. $^{1,2}$ Several factors are known to affect their colourant properties: for example, the chemical structure and concentration of the anthocyanin, the $\mathrm{pH}$ of the medium and the presence of other molecules which may act as co-pigments. In aqueous solutions, at acidic $\mathrm{pH}$ values $(\mathrm{pH}<6)$ the anthocyanins can exist as an equilibrium mixture of four different structural forms: the coloured flavylium cation $\left(\mathrm{AH}^{+}\right)$and the quinoidal bases (A), the colourless hemiacetal (B) and chalcones (C), as depicted in Scheme 1. The potential use of anthocyanins as food colourants, is thus hampered by the fact that at the physicochemical conditions required by the food industry and in the absence of other substrates they exist mainly in colourless forms. ${ }^{3}$ Moreover, under such conditions anthocyanins also undergo thermal and photochemical degradation. ${ }^{4}$

Anthocyanin co-pigmentation ${ }^{5}$ by other molecules (generally other polyphenols or flavonoids), which by themselves are colourless, seems to be one of the most promising ways to circumvent the loss of colour. However, to our knowledge, with two exceptions, ${ }^{6,7}$ no work is known about the influence of the addition of salts on the anthocyanin equilibria depicted in Scheme 1.

In both papers strong hyperchromic effects on the visible absorption band due to the flavylium cation were observed, and the authors interpreted the phenomenon as a decrease in water activity due to the anion solvation which will hinder the hydration of the flavylium form, thus displacing the equilibrium toward this form, with the consequent gain of colour.

In this work, we propose a complementary model to explain the colour enhancement of anthocyanins by salt addition, through the formation of ion-pairs between the charged flavylium cation and the anions. The thermal and photochemical stability of the ion-pairs formed by several anthocyanins [malvin (malvidin 3,5-diglucoside), oenin (malvidin 3glucoside), malvidin and 4',7-dihydroxyflavylium] is also studied.

\section{Experimental}

The natural anthocyanins (malvin and oenin) and the anthocyanidin malvidin were purchased from Extrasynthèse and used as received after purity verification by HPLC. The synthetic anthocyanin 4',7-dihydroxyflavylium chloride was kindly supplied by the group of Prof. R. Brouillard in Strasbourg. All other chemicals used were of analytical grade.

The $\mathrm{pH}$ was measured with a Metrohm $713 \mathrm{pH}$ meter. Adjustments to the desired $\mathrm{pH}$ value were accomplished by addition of a few mm $\mathrm{mm}^{3}$ of $\mathrm{NaOH}, \mathrm{HCl}$ or $\mathrm{HClO}_{4}$, except for the NMR experiments where it was adjusted with $\mathrm{NaOD}$ or $\mathrm{DCl}$.

Absorption spectra were recorded on a Perkin-Elmer Lambda 6 spectrophotometer. A constant temperature of $24^{\circ} \mathrm{C}$ was achieved using a Haake thermostatted bath.

${ }^{1} \mathrm{H}$ NMR spectra were performed on a Bruker AMX-300 spectrometer.

Light excitation at $313 \mathrm{~nm}$ was carried out by a medium pressure $\mathrm{Hg}$ lamp as previously described. ${ }^{8}$ The incident light intensity was measured by ferric oxalate actinometry. ${ }^{9}$

\section{Results and Discussion}

The addition of $\mathrm{NaClO}_{4}, \mathrm{NaCl}, \mathrm{NaI}$ or $\mathrm{NaBr}$ to previously equilibrated solutions of malvin to a final $\mathrm{pH}$ of $2.2-3.3$ results in a great hyperchromic shift of the flavylium cation visible band $\left(\lambda_{\max }=520 \mathrm{~nm}\right)$, as exemplified in Fig. 1 for the case of perchlorate addition. The effect is noticeable only upon addition of the salts in concentrations greater than $1 \mathrm{~mol} \mathrm{dm}^{-3}$, i.e. tens of thousands times the concentration of malvin. Moreover, such hyperchromism increases almost lincarly with the concentration of anion added (inset of Fig. 1).

In order to confirm that the colour enhancement is due to a displacement of the equilibria depicted in Scheme 1, we compared the ${ }^{1} \mathrm{H}$ NMR spectrum of a solution of malvin at $\mathrm{pH}$ 2.25 with one of malvin and $\mathrm{NaClO}_{4}$ at the same $\mathrm{pH}$. The spectrum of the latter showed a great increase in the peaks attributed to the flavylium cation and a decrease in those due to the hemiacetal and chalcone forms, compared with the spectrum of malvin alone. This result clearly indicates a displacement in the equilibria towards the flavylium form. Furthermore, by comparing the two spectra, an upfield shift of the peak attributed to the $2^{\prime}$ and $6^{\prime}$ protons of the flavylium cation is observed when the anion is added, as opposed to the downfield shifts observed for the remaining flavylium protons. Such an upfield shift may be due to a charge effect due to the proximity of the anion to the charged oxygen atom of the pyrylium ring which increases the shielding of the protons of the neighbouring B ring, thus displacing upfield the respective NMR peaks. ${ }^{10}$ These data also support the 